\title{
EFFECT OF DIFFUSION OF MAGNETIC PARTICLES ON THE PARAMETERS OF THE MAGNETIC FLUID SEAL: A NUMERICAL SIMULATION*
}

\author{
M.S. Krakov ${ }^{1}$, I.V. Nikiforov ${ }^{2}$ \\ 1 Belarussian National Technical University, \\ 65 Independence Ave., Minsk, 220013, Belarus \\ ${ }^{2}$ Belarussian State University, 4 Independence Square, Minsk, 220050, Belarus
}

In the paper, a motion of magnetic nanoparticles in a high gradient magnetic field and its correlation with the characteristics of the magnetic fluid seal are numerically studied. The neutral curve defining a range of parameters, where the liquid keeps fluidity, is found. It is shown that the concentration of particles during the initial time period grows linearly and then the exponent decreases to 0.5 with time. It is found that at a high enough value of magnetic field the area of close-packed particles is formed under the pole tip. Numerical simulation has shown that with some values of the parameters it is possible to decrease the magnetic particles' concentration essentially so that the basic fluid leaks out from the seal under gravity, i.e. the magnetic fluid seal fails. It has appeared that the characteristic time of the described processes depends on the properties of magnetic fluid and on the magnetic field value and has an order from several hours to several years.

1. Introduction. In magnetic fluid (MF) seals, the magnetic fluid is exposed to a high gradient magnetic field, and the energy of a magnetic particle is four orders of magnitude greater than that in a gravitational field. Therefore, the translational motion of nanoparticles in the zone of maximum magnetic field around the top of the pole tip becomes essential, and their distribution in the volume becomes non-uniform $[1,2]$.

The experiments made by S. Taketomi [2] have demonstrated that the sealing ability (the burst pressure drop $\Delta p$ ) of static seals depends on the elapsed time $t$. In the beginning, the burst pressure increases and, with reference to analytical calculations [2] for a constant gradient of magnetic field, the concentration of particles grows in the seal gap in proportion to $\sqrt{t}$. Then during some elapsed time, the burst pressure does not change. However, at the third stage it suddenly starts to grow again. While the first two stages can be explained by the increase of the particles' quantity around the top of the pole tip up to close-packed arrangement, the third one remains unclear.

There is no answer to some questions for the first two stages either. First, there arises a question of the concentration growth rate. Secondly, there is a problem of seal parameters, at which the fluid in the gap remains a fluid instead of turning into an agglomerate of close-packed particles. Thirdly, in the external part of the magnetic fluid, which is in the zone of a comparatively weak magnetic field, the concentration of particles can become so small that the magnetic field is unable to hold the basic fluid in the seal and it leaks out from the seal under gravity. The seal parameters, at which such type of failure is possible, are unknown either. Besides, it is unknown what the time of stabilization of particles' distribution in the seal is - this is one of the main problems at the analysis of its operation.

\footnotetext{
* The research results were reported at the 13th International Conference on Magnetic Fluids (ICMF-13), January 7-11, 2013, New Delhi, India.
} 
The aim of the study is to define the parameters of the magnetic fluid and MF seal, at which the latter reliably operates during the given time.

2. Governing equations. The change of the particles concentration $c$ is governed by a mass flux $\mathbf{i}$

$$
\rho \frac{\partial c}{\partial t}=-\operatorname{div} \mathbf{i}
$$

The mass flux of the magnetic particles under the action of a non-uniform magnetic field and Brownian diffusion can be written as $[3, \underline{4}]$

$$
\mathbf{i}=-\rho D \nabla c+\rho c b \mu_{0} m \nabla H
$$

with a diffusivity $D$, a mobility of particles $b$ equal to the ratio of their velocity to the driving force. For small spherical particles, their mobility is specified by the Stokes formula $b=(1 / 6) \pi \eta R$. The magnetic moment of particles is $m=M_{\mathrm{I}} V_{\mathrm{m}}$, where $M_{\mathrm{I}}$ is the saturation magnetization of a magnetite single crystal and $V_{\mathrm{m}}$ is the volume of the particle magnetic core.

Taking into account the Einstein's formula $D=k T b$, equation (1) can be written as

$$
\rho \frac{\partial c}{\partial t}=\nabla\left(\rho D \nabla c-\frac{\rho c D}{k T} \mu_{0} m \nabla H\right) .
$$

The viscosity of colloid solution of solid particles, as known, depends on their concentration $\eta=\eta_{0} f(c)$. It means that the diffusivity

$$
D=\frac{k T}{6 \pi \eta R}=\frac{k T}{6 \pi \eta_{0} R f(c)}=\frac{D_{0}}{f(c)} .
$$

Further, we will use the Vand's formula [5]

$$
f(c)=\exp \left[\frac{2.5 c+2.7 c^{2}}{1-0.609 c}\right] .
$$

Using, as scales for space coordinates, the width of the seal gap $a$, for the magnetic field its strength at the gap center $H_{0}$, for the time $a^{2} / D_{0}$, it is possible to present equation (3) in the dimensionless form

$$
\frac{\partial c}{\partial t}=\nabla\left(\frac{1}{f(c)} \nabla c-U \frac{c}{f(c)} \nabla H\right),
$$

where $U=\mu_{0} m H_{0} /(k T)$.

3. Problem geometry. It is assumed that the pole of the magnetic system has a hyperbolic form with the center of hyperbola on the surface of the shaft, and the distance between the apex of the hyperbola and the shaft is $a$. This scheme was used in $\underline{[6,7]}$.

4. Numerical method. The problem was solved in elliptical cylinder coordinates (see, for example, $\underline{[6,7]}$ )

$$
\begin{aligned}
& x=h \cos \eta \operatorname{ch} \xi \\
& y=h \sin \eta \operatorname{sh} \xi
\end{aligned}
$$

where $h=a / \cos \beta, 0 \leq \xi \leq \infty, 0 \leq \eta \leq 2 \pi$. The coordinate $x$ is calculated from the center of the hyperbola along the radius, and the $y$-coordinate along the shaft. The MF free surface was assumed coinciding with a coordinate line $\xi=$ const. 
Effect of diffusion of magnetic particles on the parameters of the magnetic fluid...

The magnetic field in these coordinates is described by the expression

$$
H=\frac{H_{0}}{\sqrt{\operatorname{sh}^{2} \xi+\sin ^{2} \xi}} .
$$

Equation (4) was solved by the numerical method of finite differences in the coordinate system (5) with the use of expression (6) for the magnetic field. The implicit conservative scheme of the second order of accuracy created by an integrointerpolation method was used. Taking into account its symmetry, the problem was solved only for $y \geq 0$. At all boundary lines of the $M F$ volume the requirement of zero mass flux was set. A grid of $251 \times 201$ nodes was used; the time step in most cases was equal to $\Delta t=10^{-4}$ (during the investigation of the initial stage, the step was equal to $\Delta t=10^{-9}$ ). To analyze the accuracy of the solution, the volume integral of the particles' concentration was controlled during calculations. In all calculations it varied eventually no more than $2.5 \%$, and such deviation has been received only after 4000000 time steps that confirms a comprehensible accuracy of the numerical analysis.

The concentration corresponding to cubic packaging was assumed to be maximum, i.e. $c_{\max }=\pi / 6$. As soon as the concentration achieved a maximum value in any node of the grid, this node was excluded from the computing process: the condition of mass flux equality to zero was transferred over the boundary line of this control volume, and this transfer guarantees that the numerical method is conscrvative.

\section{Results.}

5.1. Concentration increase with time. As the initial value, the homogeneous particles' concentration in the MF volume $c_{0}=0.12$ was set and its time history for the values of the parameter $U=0.5,5,25$ was examined. Simulation was made for the values of the geometrical parameters $\beta=45^{\circ}, y_{\max }=20$. The character of the magnetic field gradient calculated from equation (6) completely corresponds to the character of the experimental data presented in [2].

The dependence of the particle concentration increment under the top of the pole tip on the time is presented in a logarithmic scale in Fig. 1. In contrast to experimental data [2], at the initial stage, the particles' concentration grew not in proportion to the square root, but linearly to the elapsed time: $d_{c}=\left(c-c_{0}\right) \sim t$. Only after $t=10^{-5}$ the growth of the concentration slowed down, but the exponent

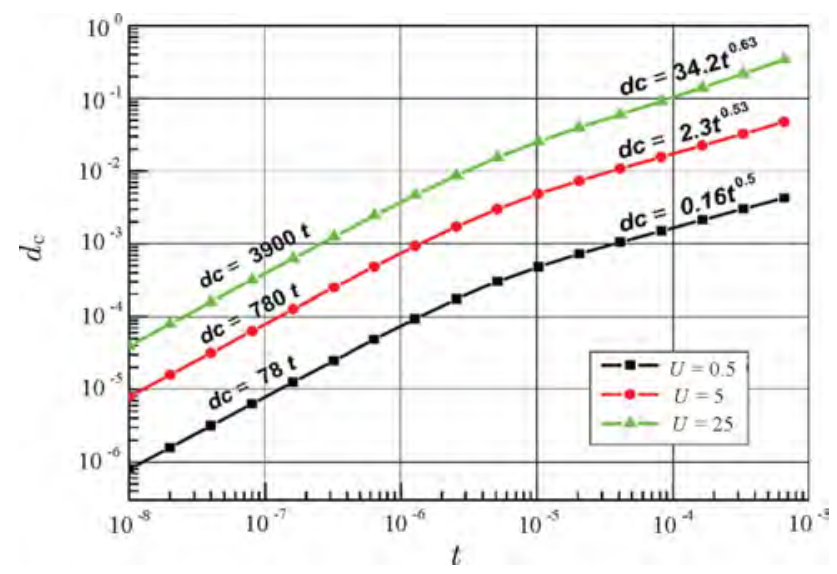

Fig. 1. Particles' concentration increase under the pole tip vs. the time; $c_{0}-0.12$. 


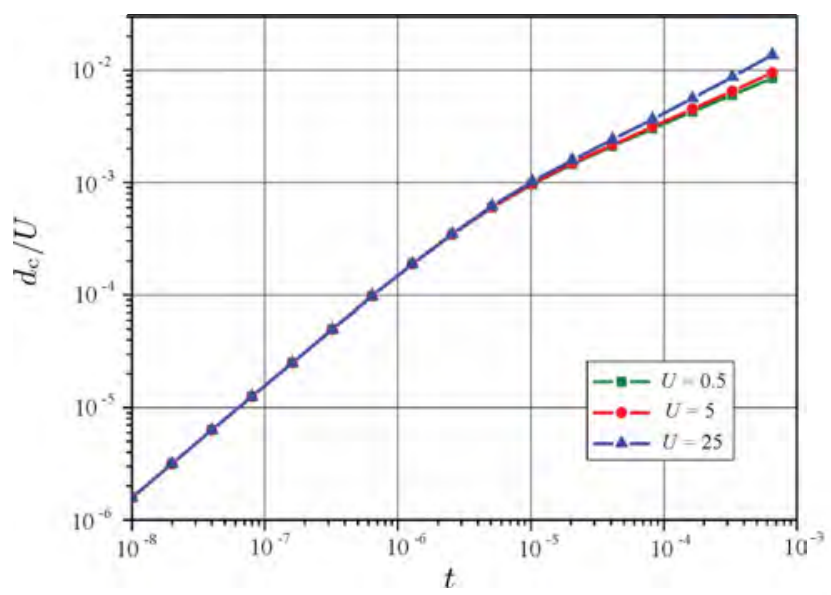

Fig. 2. Dependence of $d_{c} / U$ on the time; $c_{0}=0.12$.

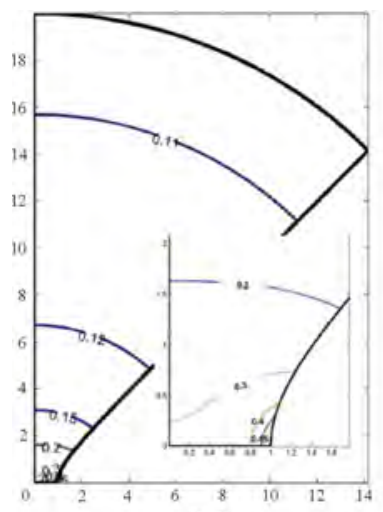

(a)

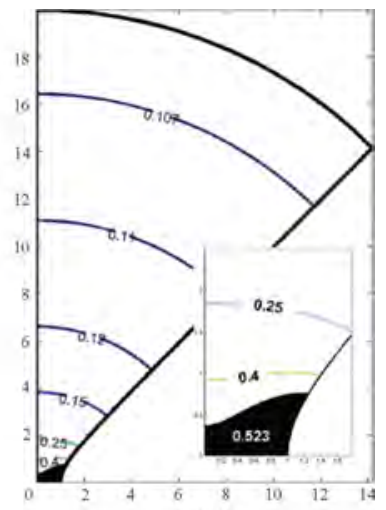

(b)

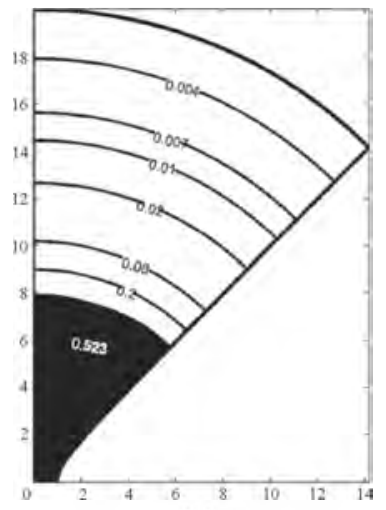

(c)

Fig. 3. Final concentration distribution: $c_{0}=0.12$. (a) $U=0.6, t=491, c_{\max }=0.517$; (b) $U=1, t=150 ;$ (c) $U=25, t=230$. The area of close-packed particles is blackcoloured.

was close to 0.5 only at small parameter $U$ values, and it was equal to 0.63 for $U=25$.

The coefficient of proportionality at the initial stage depends linearly on the parameter $U$, as presented in Fig. 2; the dependence of $d_{c} / U$ on the time is testified. As shown in Fig. 2, this curve is almost universal, and small differences for different values of the parameter $U$ probably cannot be detected experimentally.

5.2. Neutral curve. At some value $U^{*}$ of the parameter $U$ the magnetic fluid can lose the fluidic property, i.e. it ceases to be a liquid. The value $U^{*}$ must depend on the initial concentration $c_{0}$, as at $c_{0}$ close to $\pi / 6$ it obviously will be close to zero, while for very small values $c_{0}$ it will tend to infinity. The dependence $U^{*}\left(c_{0}\right)$ is important for the design of static seals, as it will allow to set such device sizes, at which the magnetic fluid in the seal does not lose its key property, i.e. fluidity.

All variants of computer simulation have been done over the range of initial concentration $c_{0}$ variation from 0.02 to $\pi / 6$. Typical variants of final, i.e. equilibrium, patterns of the concentration distribution for the case $c_{0}=0.12$ are illustrated in Fig. 3. For other values of $c_{0}$, the patterns differ only in size of the area of the close-packed particles. 


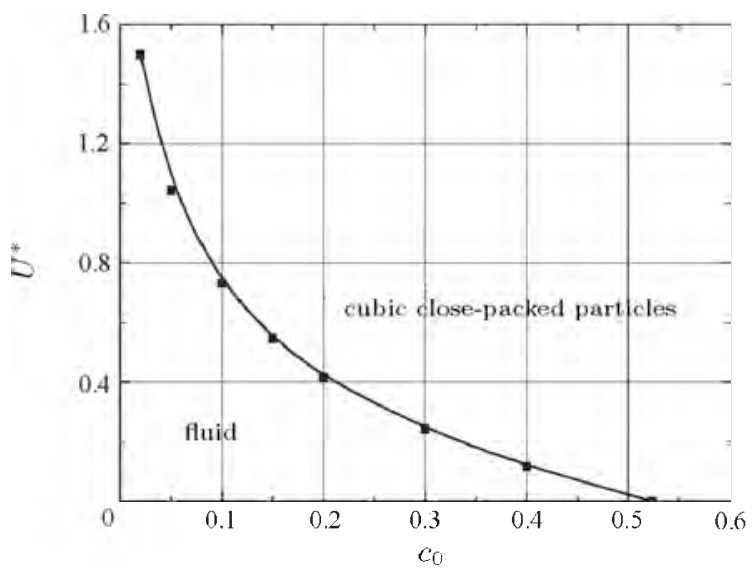

Fig. 4. The neutral curve.

As shown in Fig. 3, the maximum concentration of the particles can then appear both smaller and equal to $\pi / 6$. In the latter case, the gap can be both partially and completely overlapped by the close-packed particles. As a result of simulations, the neutral curve $U^{*}\left(c_{0}\right)$, dividing the areas $c_{\max }<\pi / 6$ and $c_{\max }-$ $\pi / 6$, has been obtained (Fig. 4 ).

In order to estimate the parameters of real magnetic fluids and burst pressure drops corresponding to the found neutral curve, we assume the particles as spherical with a complex structure: a solid particle with a diameter $d_{\mathrm{s}}$, a layer of surfactant with the thickness $l \approx 2 \mathrm{~nm}$, and an outer layer of solid particles equal to the step of the magnetite crystal lattice $h=0.84 \mathrm{~nm}$, which is non-magnetized. It means that the concentration of the magnetic phase $c_{\mathrm{m}}$ in a colloidal solution is related to the concentration $c$ by the ratio

$$
c_{\mathrm{m}}=\left(\frac{d_{\mathrm{s}}-2 h}{d_{\mathrm{s}}+2 l}\right)^{3} .
$$

The saturation magnetization $M_{\mathrm{S}}$ can be defined as $M_{\mathrm{S}}=M_{\mathrm{I}} \cdot c_{\mathrm{m}}$, where $M_{\mathrm{I}}=480 \mathrm{kA} / \mathrm{m}$ is the magnetization of crystal magnetite. In that case, on the

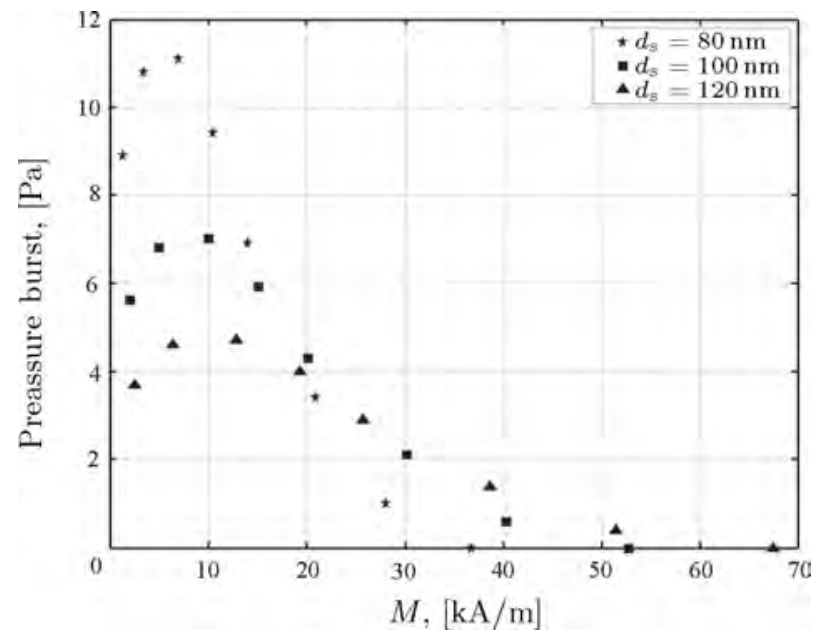

Fig. 5. The critical burst pressure drop vs. the magnetization saturation for an $\mathrm{MH}^{\mathrm{T}}$ seal with the parameters corresponding to the neutral curve. 
basis of the neutral curve, it is possible to calculate the data showing the interaction of the MF saturation magnetization and the burst pressure drop, which, as known, is determined by the expression

$$
\triangle p^{*}=\mu_{o} M\left(H^{*}\right) H^{*}
$$

With the given $d_{\mathrm{s}}$, we calculated $d_{\mathrm{m}}=d_{\mathrm{s}}-2 h$ and $d_{h}=d_{\mathrm{s}}+2 l$, then the magnetic moment $m=M_{\mathrm{I}} \pi d_{\mathrm{m}}^{3} / 6$ and $\zeta=\mu_{0} m / k T$ were calculated. After that the saturation magnetization $M_{\mathrm{S}}=M_{\mathrm{T}} c_{0}\left(d_{\mathrm{m}} / d_{h}\right)^{3}$ was calculated for the initial concentrations $c_{0}=0.02 \div 0.4$. The critical value $U^{*}$ was found from the neutral curve, and the strength of the magnetic field $H^{*}=U^{*} / \zeta$ was also found. Next, from equation (8) it was possible to define the magnetization $M=M_{\mathrm{S}} L\left(U^{*}\right)$ with the Langevin function $L$ and the burst pressure drop. The results are illustrated in Fig. 5.

As we see in Fig. 5, the neutral curve corresponds to a rather small burst pressure drop of $10 \mathrm{~Pa}$, or $10^{-4}$ of atmospheric pressure. The maximum values are typical for fluids with a saturation magnetization of $10 \mathrm{kA} / \mathrm{m}$. So the small pressure drop is determined by the fact that the absence of the area of close-packed particles is possible only at a very weak magnetic field under the pole tip of the magnetic system, at about $10 \mathrm{kA} / \mathrm{m}$.

5.3. Stabilization time of equilibrium distribution. The process of variation of the particle concentration in a magnetic fluid with time has becn analyzed. The
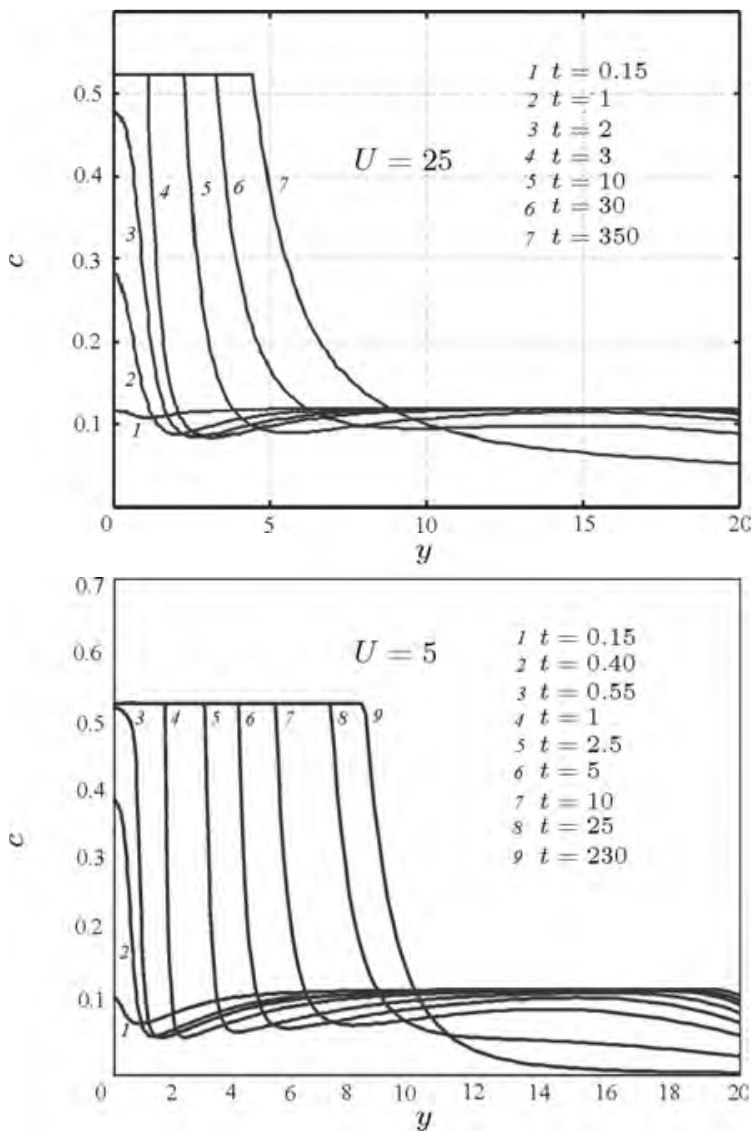

Fig. 6. Time history of the concentration distribution. $\beta=45^{\circ}, c_{0}=0.12$. 


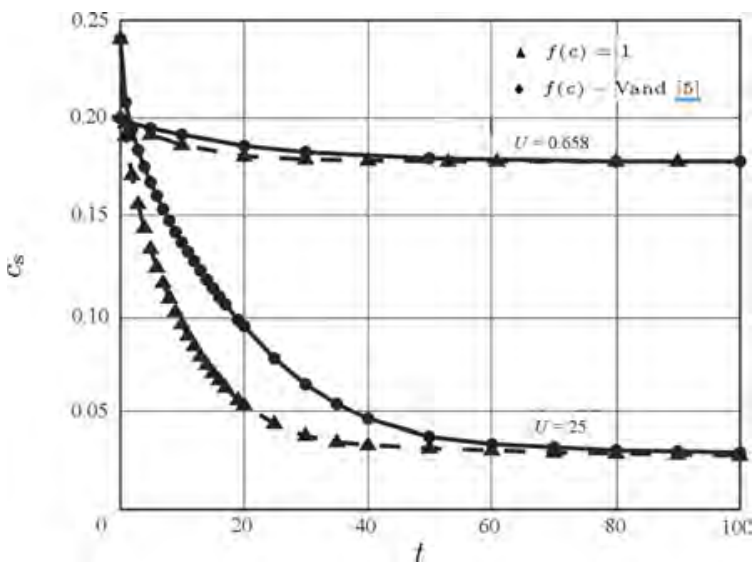

Fig. \%. Time history of the concentration at the free surface.

time history of the distribution of the particles' concentration along the shaft for $U=5$ and $U=25$ is shown in Fig. 6 .

It should be mentioned that the area of the close-packed particles appears quickly enough (as shown in Fig. 3 , with $U=25$ this time is equal to $7 \cdot 10^{-4}$ ), but the gap is completely overlapped much slower by the close-packed particles (for the same case, as shown in Fig. 3, the time equal to $l=0.55$ is required). For the whole range of initial concentrations and values of the parameter $U$, the time of the gap overlapping varied from 0.1 to 2 .

The dependences $c(t)$ at the free surface of the magnetic fluid for the essentially differing values of $c_{0}$ and $U$ are presented in Fig. 7. It could be seen that the time of stabilization of the concentration distribution lies in the range $t=60-80$ for all values of the parameters. Numerical simulation of the problem for a wide range of the parameters shows that the equilibrium time, nevertheless, depends both on the initial density and on the parameter $U$. With increasing of the initial concentration this time is slightly reducing, and with increasing of the parameter $U$ it is noticeably decreasing. But in the examined range $\left(c_{0}=0.02-0.36\right.$, $U=3.5-90)$, this time varied over the range from 23 (for $c_{0}=0.36, U=90$ ) to 300 (for $c_{0}=0.02, U=3.5$ ), remaining close to 100 for the values corresponding to real seals.

Let us estimate the scale of the dimensionless time $t_{0}-a^{2} / D_{0}$. The gap width $a$ in static seals can be varied within $a=0.2-0.6 \mathrm{~mm}$. The viscosity of the fluids used as the basis for magnetic fluids, for example, of oils, at room temperature can be in the range $0.02-0.1 \mathrm{~kg} / \mathrm{ms}$. Then the diffusivity is equal to $D_{0}=1.57 \cdot 10^{-12} \mathrm{~m}^{2} / \mathrm{s}$ for magnetite particles of $10 \mathrm{~nm}$ in size in a fluid with the viscosity $0.02 \mathrm{~kg} / \mathrm{ms}$. For a gap with $a=0.2 \mathrm{~mm}$ we obtain $t_{0}=7.1 \mathrm{hrs}$, and for a gap with $a=0.6 \mathrm{~mm}$ we have $t_{0}=64 \mathrm{hrs}$. With the fluid viscosity $0.1 \mathrm{~kg} / \mathrm{ms}$, $D_{0}=3.14 \cdot 10^{-13} \mathrm{ml}^{2} / \mathrm{s}$, and for a gap with $a=0.2 \mathrm{~mm}$ we get $t_{0}=35.3 \mathrm{hrs}$, but for $a=0.6 \mathrm{~mm}$ we get $t_{0}=318 \mathrm{hrs}$ or 13 days. As we see, the characteristic time varies in a rather wide range (from 7 to $318 \mathrm{hrs}$ ) depending on the chosen fluid and seal design.

Thus, the time of the static seal gap overlapping by the close-packed particles depends on the fluid viscosity and on the magnetic field strength in the gap and varies from several hours (that corresponds to the experimental data [2]) to 2 weelss. Accordingly, the process of final stabilization of the particles' distribution in the entire volume of the magnetic fluid, for which the characteristic time is $t \sim 100 t_{0}$, can take from one month to four years. 
Probably it is a reason of the burst pressure drop increase for the static seal after some period of its stabilization found in $[2]$ and so far having no explanation. If the magnetic field in the gap is not too strong, the final pattern displayed in Figs. $3, b$ is also analogous; after reaching the maximum value $\triangle p$ there are no reasons for its further increase. This situation corresponds to the case of the widest gap in experiments $\underline{\underline{2]}}$. However, if a large enough plug of the close-packed particles is formed in the gap, as shown in Fig. 3c, such plug behaves not as a fluid but as a ductile body.

5.4. Destruction of magnetic fluid in the static seal. Under a strong enough magnetic field and with a small initial concentration, a situation is possible when the number of particles near the MF surface during establishing of the equilibrium distribution becomes so small that the fluid practically loses its magnetic properties. As a consequence, from the volume filled with a magnetic fluid, a "tear drop" of the basic fluid will leak out under the action of gravity.

The fluid leaks out from the seal when its weight becomes more than the magnetic force keeping it: $\rho g>\mu_{0} M|\mathrm{~d} H / \mathrm{d} y|$. Let us assume that $M=M_{\mathrm{I}} c_{\mathrm{m}}$ and $c_{\mathrm{m}}$ with account of $(7)$ is related to the concentration $c$ via the ratio $c \approx$ $4 c_{\mathrm{m}}$, and also that $\mathrm{d} H / \mathrm{d} y=\left(H_{0} / a\right)(\mathrm{d} H / \mathrm{d} y)_{\mathrm{dim}}$. The dimensionless gradient of the magnetic field along the shaft $(\mathrm{d} H / \mathrm{d} y)_{\mathrm{dim}}$ is found from expression (6), and for $y=10$ it is equal to $\left|(\mathrm{d} H / \mathrm{d} y)_{\mathrm{dim}}\right|=0.00971$ and for $y=20$ we have $\left|(\mathrm{d} H / \mathrm{d} y)_{\mathrm{dim}}\right|=0.00248$. Then the above requirement is fulfilled if

$$
c<c^{*}=\frac{4 \rho g a}{\mu_{0} M_{\mathrm{I}} H_{0}} \frac{1}{|\mathrm{~d} H / \mathrm{d} y|_{\operatorname{dim}}} .
$$

For comparative analysis two cases have been chosen:

1) $a=0.2 \mathrm{~mm}, y=20$

2) $a=0.4 \mathrm{~mm}, y=10$.

As the gap width $a$ is used as a distance scale, both these cases correspond to the $4 \mathrm{~mm}$ distance from the free surface to the plane of symmetry. These two cases are good for comparison as the MF volume in both cases differs a little and critical values of the surface concentration are close $\left(c^{*} \approx 0.011\right)$. The derived relations for both investigated cases are shown in Fig. 8.

6. Conclusions. The fluid can remain fluidic in the static seal only if the magnetic field in the seal gap is small enough and the fluid magnetization is not

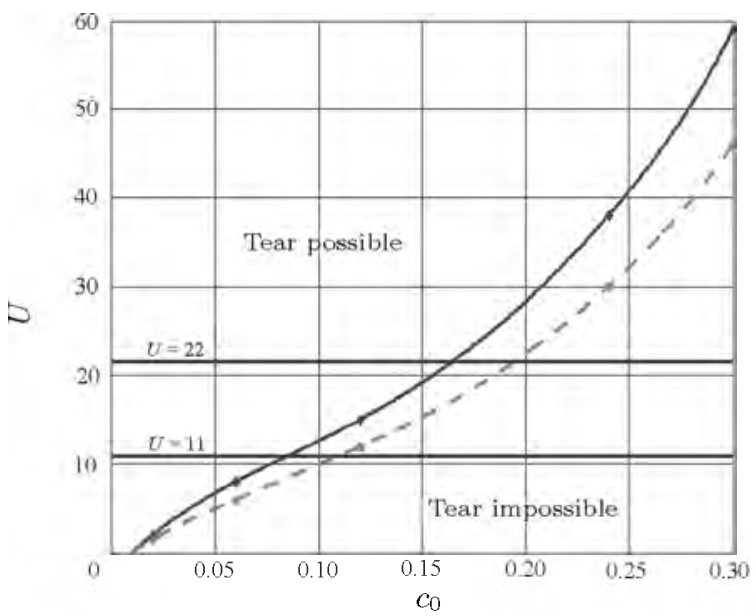

Fig. 8. Neutral curve for magnetic fluid destruction. Solid line: $a=0.2 \mathrm{~mm}$, dashed line: $a=0.4 \mathrm{~mm}$. 
too large. It has been discovered that from this point of view the magnetic fluids with a saturation magnetization of $10 \mathrm{kA} / \mathrm{m}$ are optimal.

The characteristic dimensionless time of filling the gap with close-packed particles is $t \sim 1$ that is in line with the physical time (depending on the fluid viscosity and gap width) from several hours to several weeks.

The dimensionless time of establishing of the equilibrium distribution of magnetic particles in the entire volume of magnetic fluid in the static seal in a wide range both of initial concentrations and of magnetic field values is $t \sim 100$, i.e. this process can last from a month to several years.

At small initial concentrations of particles and in a strong magnetic field, the destruction of magnetic fluid and the leakage of the basic fluid from the static seal are possible.

The analysis allows to recommend, as an optimal design, a static seal, in which the magnetic field at the gap center has a value no more than $200-300 \mathrm{kA} / \mathrm{m}$ and the magnetic fluid has a magnetization of $15 \mathrm{kA} / \mathrm{m}$ with as much as possible viscous basis.

\section{REFERENCES}

[1] B.M. Berkovski, V.F. Medvedev, M.S. Krakov. Magnetic Fluids: Engineering Applications (Oxford University Press, Oxford, United Kingdom, 1993), $243 \mathrm{p}$.

[2] S. TAKETOMI. Motion of ferrite particles under a high gradient magnetic field in a magnetic fluid shaft seal. Japanese Journal of Applied Physics, vol. 19 (1980), pp. 1929-1936.

[3] V.G. Bashtovoi, V.K. Polevikov, A.M. Algadal. The effect of diffusion processes on the statics of magnetic fluids. Proc. Nat. Acad. of Belarus, Phys.-Techn. series, no. 3 (2006), pp. 42-48.

[4] V.G. Bashtovol, V.K. Polevikov, A Supruv, A Stroots, S. BeresNEV. Influence of Brownian diffusion on statics of magnetic fluid. Maanetohydrodynamics, vol. 43 (2007), no. 1. pp. 17-25.

[5] V. VAnd. Viscosity of solutions and suspensions. 1. Theory J. Phus. Coll. Chem., vol. 52 (1948), pp. 277-299.

[6] A.N. Vislovich. V.K. Polevikov. Effect of the centrifugal and capillary forces on the free surface shape of a magnetic liquid seal. Maqnetohydrodynamics, vol. 30 (1994), no. 1, pp. 67-74.

[7] M.S. KRAKOV, I.V. Nikiforov. Influence of the meridional flow and thermomagnetic convection on characteristics of the magnetic fluid seal. Tech. Phys., vol. 56 (2011). pp. 1745-1753 (in Russ.). 